\title{
La comercialización del deporte desde la Ética de la competición deportiva
}

\author{
RAÚl FRANCISCO SEBASTIÁN SOLANES \\ Universidad de Valencia (España) \\ Raul.Sebastian@uv.es
}

\begin{abstract}
Resumen
La ética del deporte surge como una disciplina particular. Podemos encontrar dos lados radicalmente opuestos, el de aquellos que ven la comercialización del deporte como la corrupción del ideal de búsqueda de excelencia inherente a la práctica deportiva, son los partidarios de la «tesis de la corruptibilidad» y la de otro grupo, entre los que se encuentra Simon del «Hamilton College» en EEUU, que sostiene que la comercialización puede presentar un lado positivo éticamente aceptable siempre que se plantee como medio para alcanzar los valores internos en el deporte. En mi exposición abogaré por un enfoque aristotélico que entiende que la prudencia, no sólo comporta el conocimiento de los fines sino también el de los medios-fines.
\end{abstract}

Palabras clave: ética del deporte, ética de la competición, ética aristotélica, comercialización del deporte.

\section{The commercialization of the sport from Sports competition Ethics}

\begin{abstract}
Ethicals of Sports is a very particular discipline: there are many authors that try to solve problems; these problems are because many times sport becomes an object with greedy interests. Our work is giving the true orientation. On one hand, exists the groupof investigation that see sports as a corruption of the aspect relationated with it (excellency, fair play). On the other hand, Simon from Hamilton College in EEUU, suggests the study of sport in an ethical proposal if it is considered a way to internal values that sports has. My exposition will be aristothelic, considering all the points of wiev, and giving the adecuated place to the economical part.
\end{abstract}

Key words: ethic of sport, competition ethics, aristotelic ethic, commercialization of sport.

Doctorando en Filosofía por la Universidad de Valencia con una tesis sobre "Ética aplicada al deporte" dirigida por Adela Cortina. Ha sido becario de Investigación FPU (AP2006-03982) del Ministerio de Educación y Ciencia. Actualmente es becario y colegial en el Real Colegio de España en Bolonia (Italia). En la Universidad de Bolonia ha iniciado su segunda tesis doctoral en Sociología, bajo la dirección del profesor Pierpaolo Donati y que versa sobre las relaciones entre sociología y ética del deporte. Entre sus publicaciones más recientes cabe destacar: "Ética del deporte: El problema ético de las faltas estratégicas en deporte" (2009), "La ética del deporte en el contexto actual de la filosofía" (2010), "La ética del deporte en Karl-Otto Apel: Ética del discurso y compromiso trascendental claves para un deporte ético" (2011), "La ética del deporte nueva fuente de valores y deberes morales en las sociedades post-modernas" (2011).

Este trabajo se inscribe dentro del proyecto de investigación con referencia FFI200806133/FISO financiado por el Ministerio de Ciencia e Innovación. 


\section{La ética de la competición deportiva}

Dado que puede resultar chocante de entrada hablar de una «ética de la competición deportiva» debido a que la «ética del deporte» todavía es una disciplina reciente, en proceso de consolidación dentro del ámbito académico, conviene aclarar algunos datos. En primer lugar, debo decir que Robert. L. Simon, filósofo y ético del deporte en cuyo pensamiento está inspirado el presente artículo, es profesor de filosofía en el Hamilton College de EEUU, su especialidad es la ética y los valores sociales. Ha sido presidente de la «Philosophic Society for the Study of Sport», la actual «International Association for the Philosophy of Sport», es además miembro del consejo de redacción del Journal for the Philosopby of Sport en donde ha publicado un amplio número de artículos en los que ha arrojado nueva luz en materia de filosofía y ética del deporte. Entre sus principales libros publicados, puede apreciarse una evolución de su pensamiento que le ha conducido desde la sociología a la filosofía y la ética del deporte, siendo el impulsor del Internalismo ético en deporte y creador de una propuesta que él mismo ha denominado: «ética de la competición». Entre sus principales publicaciones cabe destacar Fair play: Sport, Values and Society, que ve la luz en 1991, pero también Fair Play: The Ethics of Sport, publicado en 2002 y que ya va por la segunda reedición.

Resulta obvio, que cualquier deporte profesional implica un lado competitivo del que no es posible desvincularse y que se debe tener en cuenta desde cualquier propuesta seria de ética del deporte, pero tenemos que ver cómo debe entenderse el lado competitivo del deporte, para lo que la propuesta de Simon resulte del todo pertinente, viendo en qué medida su propuesta de ética de la competición puede contribuir al correcto uso de la comercialización del deporte que no puede ser otro que el propio de una economía ética.

En ética del deporte es famoso el lema de Vince Lombarda que dice: «ganar no es lo más importante, es lo único importante»; que choca de entrada con el sabio lema del barón Pierre de Coubertin que decía: «lo más importante en la vida no es la victoria, sino la lucha; lo principal consiste no en vencer, sino en haber luchado bien».

Por regla general, se entiende que la «competición leal» es la que se realiza en base a lo establecido por las reglas constitutivas del deporte, tal como sostiene una de las principales corrientes en ética del deporte como es el Formalismo iniciado por el histórico Warren P. Fraleigh, para quien cualquier persona que cometa una trampa ya no compite en condiciones leales, por lo cual no puede aspirar a proclamarse ganador de la misma, pues ha roto la «zona de consistencia» como marco legítimo de acción dentro de la competición deportiva. 
Simon propone una pertinente y necesaria valoración moral de la función competitiva del deporte, dividiendo en dos tipos los argumentos que se dan sobre la moralidad de la competición deportiva que son: primero, los que plantean la competición deportiva en base a las consecuencias - buenas o malas- que pueda tener en la práctica, tanto referidas a los competidores, como a la sociedad en general; segundo, los que dan más importancia al carácter y valor intrínseco de la competición, sin preocuparse de las posibles consecuencias. En este último grupo es donde debe entender la propuesta de ética de la competición llevada a cabo por Simon, como muestro a continuación.

En efecto, Simon considera que aunque de entrada analizar las consecuencias (buenas o malas) de nuestra práctica en la competición deportiva pueda parecer una buena estrategia, lo cierto es que su aplicación plantea más dificultades de lo que pueda parecernos a simple vista. Por consiguiente, del hecho de que una práctica deportiva pueda tener malas consecuencias no podemos concluir que el lado competitivo en el deporte sea éticamente inaceptable, como sostienen muchos de los detractores que no aceptan la carga positiva que puede tener el horizonte competitivo en el deporte. Con todo, creo que Simon no se está dando cuenta de que además de las consecuencias malas, también puede haber buenas consecuencias que ayuden a orientar mejor nuestra acción en el deporte y que permitan hacer frente a nuestras responsabilidades como participantes.

Me explico. Uno de los principales logros de la ética del discurso defendida por Karl-Otto Apel (1998: 147ss), es concebirla como una ética de la responsabilidad, lo que supone la trasformación postmetafísica de la ética kantiana. En efecto, a diferencia del deontologismo que puede apreciarse en la propuesta de la ética kantiana, donde no se tenía en cuenta las posibles consecuencias de la aplicación del deber, en la ética del discurso se debe asumir la responsabilidad solidaria por las consecuencias y subconsecuencias a escala mundial de las acciones de los hombres, y este aspecto se debe tener muy en cuenta en el deporte, donde, como dice Cortina (2010: 45), los problemas de aplicación no son meramente problemas de estrategia - como sí ocurre en economía y política, en las que la estrategia es imprescindible- pues en el deporte se trata de orientación de la acción. Por esta razón, tener en cuenta las consecuencias - buenas o malas- de nuestras acciones puede ayudarnos a orientar nuestra acción dentro del ámbito de la competición deportiva, con lo que de entrada la posición de Simon resulta revisable en este aspecto. Además, cabe recordar que el procedimentalismo ético -que forma parte de la propuesta de la ética del discurso y que contribuye a crear un marco de dialogicidad- pese a que su principal pretensión es la 
de universalidad - por lo que trasciende la praxis concreta y el contexto concreto-, lo cierto es que también posibilita que los procedimientos se lean en la praxis concreta, ayudándonos a orientar correctamente nuestra acción en los contextos concretos en los que se desarrolla, a la vez que puede contribuir al buen encarrilamiento de la comercialización del deporte (Cortina, 2001a: 79).

Ahora bien, conviene adentrarse más a fondo en los presupuestos que forman su propuesta de ética del deporte como ética de la competición, antes de emitir cualquier juicio al respecto.

La tesis más importante que sostiene Simon desde su propuesta ética, es no plantear la competición deportiva como juegos de suma cero donde uno gana a costa de que el otro pierda. Esto implica de entrada el replanteamiento de la naturaleza de la competición deportiva desde el internalismo ético que sostiene nuestro autor, erradicando la idea de que ganar es lo único importante en deporte. Se trata de que todas las partes afectadas en la competición deportiva salgan beneficiadas, impidiendo a toda costa la tradicional división entre ganador y perdedor.

El tradicional planteamiento de la competición deportiva como juego de suma cero, donde uno gana a costa de la derrota de su adversario mostrando un comportamiento egoísta que lleva a cada participante a buscar el beneficio propio a expensas de los demás, es desestimado por el propio Simon que lo tacha de inmoral. Este ha sido uno de los principales problemas que han afectado al deporte y que en ocasión terminan convirtiendo la competición deportiva como un juego de suma cero, dejando aflorar el «espíritu de egoísmo» de los participantes implicados en la competición deportiva. Olvidando que el trato por igual a las personas es mucho más importante que el proclamarse campeón en la competición deportiva.

Ya Homero en la Iliada, había tenido el acierto de cantar tanto la victoria de Aquiles como la derrota de Héctor, sin que ninguno de ellos saliera mal parado o se considerara inferior en su calidad de vencedor o derrotado. De hecho, ambos están inscritos en la eternidad haciendo factible los versos de Píndaro que decían que «la virtud perdura en gloriosos cantos». El problema está en que como inmediatamente señala el poeta: «pero a pocos es fácil obtenerlos».

Simon se convierte en un nuevo Homero, que desde su propuesta de ética de la competición quiere erradicar la nefasta e inmoral distinción entre ganadores y perdedores, igualándolos siempre y cuando compitan buscando recíprocamente la excelencia, respetando las reglas del deporte y en condiciones de igualdad de habilidades físicas o mentales.

En efecto, desde la propuesta de ética de la competición, se considera como moralmente inaceptable enseñar a los participantes en el 
deporte que lo más importante es ganar a toda costa y utilizar cualquier medio, incluidos los ilícitos, para ganar u organizar los encuentros de forma que se enfrenten dos equipos desiguales, donde uno tiene más oportunidades y medios para ganar al otro.

Pero tampoco debemos entender la competición deportiva como una forma de autodesarrollo, tal y como quieren hacernos ver los principales detractores de la propuesta de Simon. Dichos detractores sostienen que la ética de la competición en términos de búsqueda recíproca de la excelencia a través del trabajo cooperativo, del respeto por la reglas del deporte y de la igualdad de habilidades, en el fondo, no aporta una solución seria a los problemas que surgen en el ámbito de la competición, sino que se entiende como una especie de rodeo que evita afrontar el problema de fondo, evadiéndolos y cambiándolos por la idea de que la competición nos ayuda al autodesarrollo de la excelencia, del carácter de cada competidor ${ }^{1}$. En efecto, Simon no niega que el deporte pueda ayudarnos al autodesarrollo personal, de hecho, recuerda al respecto la posición de Harry Edward, para quien los deportes no tienen como principal objetivo la construcción del carácter de los que participan, aunque pueden contribuir al desarrollo de los trazos preexistentes del carácter de los participantes. Con todo, a Simon le parece muy difícil establecer si la participación en el deporte de competición favorece o no el desarrollo del carácter y de los elementos deseables del mismo, por lo que niega las acusaciones de sus detractores que confunden su ética de la competición con la defensa encubierta de un ideal no competitivo de autodesarrollo en el deporte.

Simon adopta una doble defensa frente a los críticos de su propuesta de ética de la competición, que abogan que en el fondo dicha propuesta no se enfrenta de cara a los problemas que ofrece la competición en el deporte sino que los evade concibiendo dicha competición como una forma encubierta de autodesarrollo personal y contra los que conciben la competición como un juego de suma cero, donde una parte se beneficia a costa de la perdida de la otra.

En primer lugar, Simon entiende que la competición deportiva no debe entenderse como un planteamiento que nos conduce a una actitud egoísta, como la que puede aflorar si planteamos la competición como

Viktor E. Frankl (1982: 103-112) desde su propuesta de logoterapia, entiende precisamente que el deporte nos ayuda a evitar que se convierta en una práctica cuyo principal objetivo es el de derrotar a mis oponentes y proclamarme campeón del encuentro. Frankl propone entender nuestra participación en el deporte como una competición con uno mismo, en donde vemos hasta donde podemos llegar con nuestra práctica en el deporte. Este tipo de propuestas no deben confundirse con la que hace Simon. 
un «juego de suma cero», donde el afán por ganar derrotando a nuestro oponente nos lleva a buscar nuestro propio beneficio sin importarnos el beneficio mutuo que favorece a las dos partes implicadas. La propuesta de ética de la competición de Simon, supera los problemas de este «espíritu de egoísmo» y entiende que gane quien gane en deporte, ambas partes salen beneficiadas mutuamente pues si han participado bien, mostrando lo mejor de sí, esto les permitirá encajar bien los retos que les surjan en un futuro fomentando la búsqueda recíproca de la excelencia moral en deporte. En segundo lugar, la propuesta de Simon permite ver al oponente no como un rival al que hay que superar a como dé lugar con tal de proclamarse vencedor del encuentro. Para Simon debemos ver a nuestro oponente como alguien del que puedo aprender y que puede ayudarnos a crecer en la excelencia, mejorando los posibles defectos que podemos tener en la competición y cooperando con él en la búsqueda de la excelencia para que ambas partes salgan beneficiadas del encuentro y motivadas en seguir cooperando en excelencia.

Luego, la competición deportiva distingue entre el esfuerzo que se realiza para la mejora personal de nuestras habilidades físicas y mentales, donde nuestro oponente puede contribuir a dicha mejora, con el esfuerzo cooperativo que realizamos para cumplir un reto con nuestro rival. Esto, por supuesto, no convierte su propuesta de ética de la competición en una forma de evasión o defensa encubierta del ideal no competitivo de autodesarrollo en el deporte, como quieren ver los principales críticos de Simon. En realidad, Simon piensa que se evitan los problemas que acarrea el exceso de competitividad en el deporte, pues se trata de una competición cooperativa en la búsqueda recíproca de la excelencia donde todos los participantes consideran que es más importante la cooperación que competir para proclamarse vencedores.

La buena competición deportiva presupone un esfuerzo cooperativo por parte de todos los participantes que convierte la competición en una nueva forma de desafío donde cada uno saca lo mejor en cuestión de habilidades físicas y mentales buscando recíprocamente un modelo de excelencia que beneficia a todos y no sólo a una parte como ocurre con los juegos de suma cero evitando que aflore cualquier tipo de comportamiento antideportivo, o al menos minorando este tipo de comportamientos que en la mayoría de ocasiones brotan de un afán incontrolado de egoísmo personal de los participantes que sólo ven los bienes externos en el deporte olvidando que éstos son medios, pues los verdaderos fines del deporte están más en consonancia con los bienes internos o con el valor interno de la práctica deportiva. Se trata de una especie de contrato en donde las partes implicadas están de acuerdo en competir según el respeto a las reglas constitutivas y de manera 
equitativa, siempre y cuando este presupuesto sea acepado voluntariamente como parte de la búsqueda mutua de la excelencia.

El objetivo de la buena competición deportiva es la búsqueda mutua de la excelencia que sólo puede alcanzarse a través del trabajo cooperativo y el respeto a las reglas del deporte fomentando la equidad en todo momento y cuya consecuencia más inmediata es que todos los participantes salen ganando aunque sea uno quien finalmente obtenga la victoria oficial, y salen ganando por que han sacado lo mejor de sí mismos como competidores mostrando sus mejores habilidades físicas y mentales, nunca por el lucro o la ganancia económica, que en todo caso debe entenderse siempre como medio para que el deporte siga subsistiendo.

La ética de la competición entiende que la competición deportiva es un esfuerzo cooperativo por parte de los participantes que les ayuda a generar una mejora en el desafío, compitiendo codo a codo con el oponente y sacando conjuntamente lo mejor de sus habilidades físicas y mentales, además de motivarles para seguir teniendo este tipo de comportamiento en el futuro. Se entiende que el principal objetivo de la competición es que todos sus participantes busquen recíprocamente la excelencia moral en el deporte y los bienes internos en él, preocupándose por cumplir con las reglas del deporte y respetando la igualdad de trato y oportunidades en proclamarse campeón del encuentro, sin que el hecho de obtener la victoria sea lo más importante en la competición. De este modo evitamos convertir la competición en un juego de suma cero, que haga aflorar un «espíritu de egoísmo» en los participantes motivándolos a competir buscando el beneficio individual y extrínseco. Pero también nos ayuda a evitar caer en el error de que desde esta propuesta en el fondo se está haciendo una defensa encubierta del ideal no competitivo de autodesarrollo, pues se entiende que el crecimiento principal en el deporte no tiene por qué ser el autodesarrollo sino el trabajo en equipo.

Desde la perspectiva de la ética de la competición, ganar no es necesariamente un signo de éxito competitivo y perder no es muestra de un fracaso. Ganar no lo es todo, pero sigue siendo algo, pues el aspecto competitivo siempre permanece presente. La clave reside en los entrenadores quienes tienen que saber equilibrar y mostrar en los entrenamientos dónde reside el verdadero énfasis, pues los efectos nocivos están en el exceso de énfasis en lograr la victoria, así como en la falta de motivación por obtenerla. Lo que no puede considerarse en modo alguno es que la finalidad de la competición deportiva sea la pura ganancia económica. 


\section{La comercialización del deporte desde la ética de la competición de Robert L. Simon}

La comercialización del deporte es una de las últimas preocupaciones que van apareciendo en las obras más recientes de Simon y que éste intenta analizar desde su propuesta de ética de la competición deportiva que cada vez está más perfeccionada, consolidada y que abarca más ámbitos de aplicación de la práctica deportiva.

En efecto, uno de los campos de estudio más importante en la ética del deporte es el de la comercialización de la práctica deportiva, especialmente del deporte de alta competición. La comercialización del deporte tiene como consecuencia más inmediata convertir el deporte, que en principio tiene que ver con el ocio, en un negocio. De entrada esto parece etimológicamente ilógico, pues la raíz latina «nec» + «otium», se refiere a lo que no tiene que ver con el ocio. La pregunta por tanto es cómo y porqué ha ocurrido que algo como el deporte que se vincula desde sus orígenes al ocio, ha devenido en negocio.

Aunque prima facie, podamos ver la comercialización del deporte como algo negativo o contrario a la ética, lo cierto es que dicha comercialización ha sido abordada desde dos perspectivas: una negativa cuyos partidarios se engloban dentro de lo que se ha denominado «corruption thesis» y otra positiva como el propio Simon lo sabe plasmar en su argumentación.

En efecto, el deporte de alta competición puede ser el mejor vehículo de transmisión de valores morales -que, según Simon, son inherentes a la misma práctica deportiva-, los cuales pueden ayudar a la búsqueda recíproca de la excelencia a través del cultivo de valores tan importantes como la dedicación o la disciplina, añadiendo la equidad y el respeto a las reglas constitutivas. El deporte puede, además, transmitirnos momentos de gran belleza estética, haciendo que el suspenso por el resultado final de la competición esté suspendido en el aire hasta el último momento y por tanto haciendo que la competición deportiva adquiera momentos de emoción y drama insospechados. Por desgracia, la propia popularidad y prestigio que posee el deporte se convierte en su punto débil, en su "talón de Aquiles», pues esta capacidad de convocatoria que tiene el deporte de élite, lo convierte en un objeto muy atractivo para el mercado que en la mayoría de casos, convierte las competiciones deportivas en eventos muy rentables y que devienen en una mercancía que tiene que venderse, en un negocio cuyo éxito está garantizado, debido a la demanda existente y que por tanto va a dar muchos beneficios a todo aquel que quiera sacarle rentabilidad. 
Aunque como señala acertadamente Mieth (1989) al abordar el problema de la comercialización del deporte, lo cierto es que los medios de comunicación han favorecido la conversión del deporte de élite en un objeto rentable, generando peligrosos vínculos de dependencia entre el sistema «deporte» y el sistema «medios de comunicación» que arroja al primero a los intereses puramente económicos.

Por su parte, Simon hace en su exposición una clara distinción entre el lado negativo que puede mostrar la comercialización del deporte como defiende la denominada «tesis de la corrupción» y un lado positivo de la comercialización que la convierte en éticamente aceptable siempre y cuando se la conciba como medio para alcanzar los valores internos a la práctica deportiva imprescindibles para que la competición se desarrolle desde la excelencia y que implica además la observancia de las reglas constitutivas y de la equidad (tal y como va a defender Simon). En esta línea voy a dividir este apartado en dos, uno referido a la «tesis de la corrupción» (corrumption thesis) y otro referido a la defensa del lado positivo que puede presentar la comercialización del deporte siempre y cuando se tengan claros cuáles son los medios y fines que debe perseguir la práctica deportiva y que obviamente tienen que ver con los valores internos y con la búsqueda recíproca de la excelencia moral, desde el respeto a las reglas y a la equidad entre los competidores que es lo que verdaderamente constituye el núcleo de la propuesta de ética del deporte como ética de la competición que defiende Simon.

\section{1. "Corruption thesis»: el lado negativo de la comercialización del deporte}

Para Simon, decir corrupción se refiere a un tipo de descomposición o a una caída del propósito original y noble de una práctica (Simon, 2004: 172). Aplicada al caso de la práctica deportiva la corrupción se referiría a que la competición deportiva ha olvidado los valores fundamentales para el deporte, inherentes a él, que posibilitan la búsqueda recíproca de la excelencia y que garantiza que los participantes cumplen con las reglas constitutivas y compiten en equidad. Dicha corrupción se debe a una causa externa que hace que el deporte equivoque el camino, como puede ser la comercialización, es decir, la ganancia lucrativa de dinero gracias a las competiciones deportivas, lo cual hace que el fin del deporte no sea la búsqueda recíproca de la excelencia junto con los valores internos a la práctica tales como son el esfuerzo o la disciplina, sino que los participantes y todo el grupo de afectados se sienta motivado a ganar el mayor rendimiento económico de su participación, directa o indirecta. 
Existe una tendencia en ética del deporte de la que Simon se hace eco en su exposición, pero de la que se quiere alejar pues entiende que la base de su planteamiento es algo exagerado y radical, ya que sólo atiende a un lado de la comercialización del deporte, que es el lado negativo y no tiene en cuenta que dicha comercialización puede tener un lado positivo. Me refiero a la «tesis de la corrupción» (corruption thesis).

En efecto, según la «tesis de la corrupción», la comercialización del deporte trasforma la práctica deportiva en un producto que puede ser comprado o vendido obteniendo múltiples beneficios económicos, lo que tiene como consecuencia más inmediata la corrupción del verdadero fin del deporte que no puede ser la ganancia lucrativa, el mayor beneficio económico.

Al respecto, William Morgan (1994: 128) sostiene que la comercialización de la práctica deportiva instala los valores de mercado en el deporte, tales como la búsqueda de dinero, la fama, la rentabilidad de las inversiones hechas en los diversos acontecimientos deportivos, dejando de lado los verdaderos fines del deporte que nada tienen de los valores de mercado ni de la economía capitalista.

Los partidarios de la «tesis de la corrupción», no sólo sostienen que la comercialización del deporte corrompe los fines de éste, sino que lo convierte en un instrumento para el logro de los bienes externos a la práctica deportiva, como son la ganancia económica o la fama.

Para Simon los presupuestos de la denominada «tesis de la corrupción» son una respuesta demasiado radical y estricta como para ser aceptada en su totalidad, por lo que requiere ser tomada en consideración analizando en profundidad todos los detalles.

A mí se me ocurren diversos puntos en donde veo claro por qué Simon no puede aceptar la tesis de la corrupción del deporte desde su postura de ética de la competición. En primer lugar, creo que Simon no puede aceptarla pues la tesis de la corrupción se ofrece como una nueva manifestación de reduccionismo socio-económico, al considerar que dicha comercialización instala los valores de mercado en el deporte, tal y como señala Morgan. En este sentido, Simon se opone a la visión reduccionista del deporte, que defiende que en la práctica deportiva se aprecian los valores imperantes de la sociedad. Esto es rechazado por Simon, abogando a favor de que la práctica deportiva tiene sus propios valores internos, tales como la dedicación, el esfuerzo y la disciplina; persigue sus propios fines como es la búsqueda recíproca de la excelencia, lo cual implica la observancia de las reglas constitutivas y la equidad en la competición, siguiendo en este punto la misma concepción de práctica que emplea Alasdair MacIntyre (1981: 175). Decir que la comercialización traslada los valores de mercado a la práctica deportiva 
constituye una afirmación poco acertada que Simon no puede aceptar. Es cierto que el deporte puede convertirse en un rentable objeto de mercado, pero sin que por ello se instalen en el deporte los valores del mercado, ya que la práctica deportiva tiene sus propios valores internos que están por encima de los valores imperantes en cualquier sociedad.

Hablar del poder corruptor que ejerce la comercialización en el deporte se refiere a la mala práctica de ciertas personas pertenecientes al mundo del deporte que guiados por fines lucrativos pervierten la práctica, pero no pervierten la naturaleza misma del deporte que se caracteriza por esa moral interna, y por la búsqueda recíproca de la excelencia a partir del respeto por las reglas y por la equidad como señala Simon.

Pero hay otro aspecto que se sigue de la «tesis de la corrupción» que es analizado por Simon y que afecta a los espectadores estadounidenses.

James Michener (1976: 17) sugiere que el gran problema de EEUU es que son una nación de espectadores antes que de participantes. En efecto, el gran problema que puede presentar el deporte estadounidense, y que los partidarios de la «tesis de la corrupción» achacan a la comercialización del deporte, es que los espectadores se dejan arrastrar por un exceso de "partidismo», de filiación y supuesta lealtad a su equipo, deseando por encima de todo que gane, por lo que se genera una cierta animadversión hacia el equipo rival, llegando a despreciar su buen juego, llegando a no reconocer el mérito y la dignidad del adversario, pues lo único que les interesa es que su equipo gane. Este partidismo genera en muchas ocasiones un comportamiento agresivo que se manifiesta en una violencia verbal en ocasiones desmedida hacia los rivales, que también puede culminar en una violencia física, bien hacia los propios deportistas o bien hacia los otros fans del equipo oponente que asisten al partido.

El problema de fondo es que a base de asistir de manera directa o indirecta a los encuentros deportivos, los espectadores no han generado una actitud crítica, sino que el exceso de partidismo les lleva a este tipo de conducta destructiva y antideportiva que se ve acrecentada por la comercialización del deporte, ya que los espectadores que pagan por asistir a una competición deportiva —donde su equipo se puede jugar la victoria de algún campeonato- quieren sacar el mayor rendimiento del dinero que han invertido para asistir de manera directa a la competición. Por ello, no es de extrañar que se sientan defraudados e incluso estafados si su equipo pierde. Obviamente, Simon no acepta esta segunda postura que sostienen los partidarios de la «tesis de la corrupción», pues se adhiere a la postura sostenida por Christopher Lasch, quien defiende que los espectadores no destruyen el valor del deporte e incluso su asistencia lo puede hacer más completo, pues una de las genialidades del deporte 
contemporáneo es que a base de buen hacer, de mostrar que lo importante es la búsqueda recíproca de la excelencia y el cumplimiento de las reglas constitutivas del deporte, puede producir que los espectadores desarrollen un juicio crítico que les convierta en personas virtuosas que valoran más el buen juego que la victoria, evitando que se conviertan en masa borreguil (Simon, 2004: 176).

Por ello, apreciar una buena competición en el transcurso de la misma requiere de inteligencia, observación y capacidad crítica, además de la aplicación de las normas de la excelencia, sin que la comercialización del deporte se vea como la principal responsable del comportamiento violento y antideportivo que demuestran algunos participantes.

No obstante, creo que la falta de capacidad crítica que en la mayoría de los casos muestran los espectadores que asiste de manera directa a las competiciones deportivas no tiene por qué ser consecuencia de la comercialización del deporte, sino más bien puede ser consecuencia del Estado de Bienestar. Me explico. Como acertadamente señala Adela Cortina, el Estado de Bienestar surge a finales del siglo XIX impulsado por Otto von Bismarck, a quien debemos la aprobación por primera vez en la historia de una serie de medidas sociales, como fueron el seguro de enfermedad, el seguro contra accidentes laborales, o las pensiones para la vejez. En este sentido, el Estado asume por primera vez funciones sociales, pues hasta entonces sólo había tenido funciones políticas. Todo esto en principio fue un avance en política social si tenemos en mente las penosas condiciones de los trabajadores de la época. Sin embargo, con el transcurso del tiempo el Estado del Bienestar pasa a convertirse en un Estado paternalista, en donde se aprecia una sobre protección que ha provocado que se le llame coloquialmente como "papá Estado» o en palabras de Drucker «megaestado», en donde se considera a sí mismo como el hacedor más adecuado para llevar a cabo todas las tareas sociales y solucionar todos los problemas de carácter social (Drucker, 1998: 127-129). Al final, esto tiene una nefasta consecuencia para la ciudadanía, pues se generan ciudadanos heterónomos, que acaban persuadiéndose de su heteronomía y asumiendo la vida política, económica y social con una actitud de «dependencia pasiva» propia de un incompetente básico. Se trata de un ciudadano criticón que no es lo mismo que crítico, pasivo, apático y mediocre del que se aleja todo pensamiento de libre iniciativa y de creatividad propia (Cortina, 2001b: 65-96).

Esto, a mi juicio, puede explicar en buena medida la actitud de los espectadores estadounidenses y de otros puntos de la «aldea global» junto con su comportamiento agresivo y criticón cuando su equipo 
pierde, lo cual no tiene por qué ser consecuencia de la comercialización del deporte - como bien aclara Simon- sino que quizás puede ser consecuencia - creo yo- de la crisis actual del Estado del Bienestar, que ha devenido en «megaestado» o en «Estado paternalista» y «electorero», que forma una ciudadanía criticona, pasiva y muy alejada de asumir responsabilidades ciudadanas y que a la primera de cambio no tiene reparo en recurrir a todo tipo de violencia verbal o física sin medir las consecuencias de su acción.

En definitiva, parece que la comercialización del deporte no sólo tiene un lado negativo - sin que por ello se niegue este lado- ni tampoco podemos achacar los brotes de violencia o de perversión que surgen en torno al deporte como consecuencia de dicha comercialización, ya que pueden ser causa de otros efectos como es la ciudadanía pasiva consecuencia directa del paternalismo que irradia el decadente Estado del Bienestar o de la crisis de valores humanos que muestra un analfabetismo emocional de la ciudadanía incapaz de compadecerse del dolor ajeno e inconsciente del dolor que puede causar a través de un comportamiento violento, como los que por desgracia surgen con más frecuencia en torno a los acontecimientos deportivos. Por esta razón, Simon aboga por un lado positivo de la comercialización del deporte.

\subsection{E1 lado positivo de la comercialización del deporte: la originalidad de Simon}

Normalmente la comercialización del deporte, se aborda desde una postura negativa, como muestran los partidarios de la «corruption thesis», que sólo destacan su lado negativo, olvidando que los deportes también necesitan de financiación económica, no sólo para pagar a los deportistas, sino a todo el personal que engloba el club deportivo y que va desde los entrenadores hasta el personal de limpieza que se ocupa del buen estado de todas las instalaciones.

Simon se aleja de los presupuestos que sostienen los partidarios de la «corruption thesis», precisamente porque éstos sólo se centran en el lado negativo de la comercialización y pasan por alto su lado positivo.

Para llevar a cabo su propósito, Simon asume la distinción entre bienes internos y bienes externos a la práctica deportiva que muestra cómo nuestro filósofo está influido en este punto por la propuesta de MacIntyre, aunque lo curioso es que no le cita en ningún momento. Esta influencia la podemos apreciar cuando Simon habla de bienes internos a la práctica deportiva. Recordemos que para MacIntyre se hace necesario distinguir entre bienes internos y bienes externos a la práctica, defendiendo la importancia de adquirir los bienes internos antes que los 
externos. Otro importante elemento que prueba la impronta de MacIntyre en Simon, es que para este último, igual que para el autor de After virtue, además de buscar los valores o bienes internos a la práctica deportiva, también es imprescindible que se cumpla con las reglas características de ese deporte en cuestión, si es que queremos que nuestra práctica sea éticamente aceptable y persiga los modelos de excelencia y de equidad que se desprenden de ella. En efecto, dichos bienes internos a la práctica lo son por dos razones: la primera es que se encuentran internos en cualquier tipo de prácticas como puedan ser el fútbol, o el ajedrez; y segundo, porque sólo pueden identificarse y reconocerse participando en este tipo de práctica, desde dentro, no desde fuera (MacIntyre, 1981: 176).

Por otra parte, la definición de práctica dada por MacIntyre, decía que en toda práctica, además de los bienes internos a ésta, conllevaba modelos de excelencia y obediencia a normas (MacIntyre, 1981: 236). También el propio Simon entiende que junto con la moral interna y junto con los valores internos en el deporte, su ética de la competición exige ante todo que los competidores respeten la capacidad de competir según las reglas de su deporte, respetando a su vez al adversario, siendo este tipo de proceder, lo que en el fondo constituye que nuestra práctica sea excelente y equitativa.

En relación a la postura que mantiene Simon con la visión positiva de la comercialización en el deporte, puede apreciarse la presencia de la distinción entre bienes internos y bienes externos a la práctica deportiva como núcleo principal para abordar el problema de la comercialización del deporte. Como he señalado, en este punto creo firmemente que Simon está muy influenciado por el pensamiento de MacIntyre, aunque la referencia a este autor no se haga explícita, pues permanece latente, oculta, pero es una realidad en toda la argumentación de Simon.

Para Simon, en la práctica deportiva existen unos bienes externos tales como la salud, la diversión, la fama y la riqueza. Junto a éstos, existen otros bienes internos que no pueden entenderse con independencia de dicha práctica, como muestra el ejemplo del «bome run» que es ininteligible fuera de la práctica y de las normas del béisbol ${ }^{2}$, o la elegancia que puede tener una combinación ganadora en ajedrez, que sería incomprensible si no se tiene conocimiento de las reglas y la estrategia que caracteriza el ajedrez.

2 En términos del béisbol, el «home run», se da cuando el bateador hace contacto con la pelota de una manera que le permite recorrer las bases y anotar una carrera, en la misma jugada, sin que se registre ningún otro error de la defensa. 
El problema de la comercialización del deporte según Simon, es que en la mayoría de los casos los deportistas sólo ven, o sólo se interesan por los bienes externos a la práctica deportiva, como son el ganar dinero o la fama que les reporta ser deportistas de élite, pero no sólo ocurre esto con los deportistas, sino también con los empresarios y demás miembros del mundo deportivo, tales como los entrenadores que pueden convertir el deporte en un negocio, en algo que tiene poco que ver con el ocio, al que en principio se vinculaba el deporte.

Este tipo de inclinaciones hacia los bienes externos que no sólo provienen de los deportistas sino de los otros «grupos de afectados» en el deporte, pueden hacer que cada uno persiga sus propias metas, olvidando que forma parte de una unidad común a la que llamamos equipo y en donde se presupone la cooperación para alcanzar las metas propuestas y el beneficio mutuo propio de la camaradería. En este punto, de nuevo, puede apreciarse la influencia del pensamiento de MacIntyre en Simon, dado que para MacIntyre en las relaciones sociales es imprescindible cooperar con los demás para alcanzar los bienes comunes a la práctica, lo que implica de ante mano una cierta comprensión compartida de posibilidades presentes y futuras, pues la independencia — dirá MacIntyre- supone la dependencia con los demás, con quienes tenemos que cooperar si queremos alcanzar los bienes comunes y la excelencia en nuestra práctica (MacIntyre, 2001: 92). Por esta razón, considero acertada y pertinente la propuesta de ética hermenéutica elaborada por Jesús Conill, el cual defiende que «compartimos el deseo de lo justo y nos sentimos afectados por un sentimiento de pertenencia que nos vincula al otro» (Conill, 2006: 179).

La trasformación del deporte de élite en una «mercancía», en un producto rentable, no presenta peligros reales para una ética del deporte, según nos aclara Simon. Para evitar contratiempos cree que antes de realizar un análisis lógico de los efectos perversos de la comercialización del deporte resulta más rentable considerar qué principios morales deben aplicarse a los deportes profesionales, aceptando que debe haber un marco ético que regule los beneficios oportunos que puede tener la comercialización en el deporte.

Con todo, creo que la exposición de Simon no deja del todo claro la importancia de defender una relación de interdependencia o complementariedad entre los bienes internos a la práctica y los bienes externos. En efecto, los bienes internos a la práctica tienen prioridad y son los que más nos aproximan a los modelos de excelencia, junto a la observancia a las reglas y la equidad que se desprenden de la práctica deportiva. Pero hay que valorar a su vez la importancia de los bienes externos y de las motivaciones externas a la práctica que también constituyen un excelente 
medio para adquirir una práctica excelente. El único inconveniente consiste en que cuando los bienes externos - me refiero a la ganancia económica, la fama, entre otros similares- se convierten en fines en lugar de medios, es entonces cuando la comercialización del deporte puede convertirse en un problema y puede presentar una faceta negativa, que convierta al deporte en un mero producto de mercado, en un negocio lucrativo y rentable, tanto para los empresarios, entrenadores y deportistas. Es preciso en este punto recordar que el propio Aristóteles entiende que la prudencia, no sólo comporta el conocimiento de los fines sino también el de los medios (Moncho, 1972: 93). En efecto, si tenemos en cuenta la opinión de muchos de los estudiosos de Aristóteles, hay que ver que en el conocimiento moral está implicado esencialmente un doble acto de cognición, que se centra en la relación fines-medios (Monan, 1959: 114).

En el caso de Simon creo que se tendría que dejar más claro qué papel tienen los bienes externos a la práctica deportiva en relación a los verdaderos fines de dicha práctica, pues quien busca los fines persigue los medios. Creo que para plantear la comercialización del deporte como algo positivo desde el punto de vista ético, es preciso considerar el importante papel de los bienes y motivaciones externos a la práctica, siempre que se los vea como medios y no como fines que nos permitan alcanzar los verdaderos fines del deporte que tiene que ver con los bienes y valores internos en el deporte, con ello sería preciso hablar de «compromiso trascendental», como lo hace Apel, cuando aborda desde la ética del discurso el fenómeno deportivo.

Lo que sí debemos reconocer en la propuesta de Simon es la reevaluación de la «corruption thesis» en el deporte. Me explico. Puede comprobarse que existen dos posiciones a la hora de afrontar el estudio crítico de la comercialización del deporte en clave ética, la de aquellos que la plantean desde la «corruption thesis» y la ven como algo negativo, pues entienden que la comercialización del deporte amenaza la búsqueda de la excelencia moral y los valores internos, en primer lugar, porque si reducimos las habilidades de un deporte, acomodándolas a aquello que hace de los eventos deportivos más entretenidos de cara al público de masa, buscando que éste salga satisfecho y vuelva a repetir gastando dinero para ello; o en segundo lugar, si la comercialización permite a los clubes más ricos - que obtienen más ganancias- la dotación de nuevas tecnologías que les permita aumentar el rendimiento y ponerse por delante de aquellos equipos más pobres que no disponen de capital para invertirlo en estas tecnologías, provocaría un grave problema de equidad, de igualdad de oportunidades a la hora de competir y optar por la victoria. 
Sin embargo, Simon nos ofrece otra visión de la comercialización del deporte en clave positiva que puede alcanzarse siempre y cuando se siga un equilibrio y una gobernanza adecuada por parte de los que están en la capa más alta de la institución deportiva. La práctica deportiva tiene el potencial de reducir los conflictos entre la comercialización y la integridad de la competición deportiva. El deporte de élite tiene el potencial de jugar un importante papel social, lo que le falta añadir a Simon es que puede tenerlo siempre y cuando se mantenga un equilibrio ético entre medios-fines, sin olvidar que el fin de la práctica deportiva sea la consecución de la excelencia moral a través de cumplimiento de las reglas constitutivas y de la equidad y que los bienes externos pueden constituir un buen medio que nos permita alcanzar los verdaderos fines del deporte y que a mi entender sólo se puede conseguir a través de un compromiso trascendental que debe asumir cada participante en el deporte.

\section{Responsabilidad social de la empresa deportiva}

Uno de los aspectos más novedosos de la propuesta de ética de la competición de Simon es el de la responsabilidad social de la empresa deportiva, que aunque es un tema cuyo tratamiento serio nos llevaría el trabajo de una tesis doctoral, lo cierto es que conviene traerlo a colación desde la propuesta Internalista de Simon y añadiendo la perspectiva de la ética del discurso en su vertiente aplicada a la economía y la empresa, unido al denominado «Stakeholders capitalism» o capitalismo de los afectados, en virtud del cual una empresa ética - como lo debe de ser la empresa deportiva - debe tener en cuenta a todos los grupos de afectados, y atender dialógicamente todos sus intereses con la pretensión de universalizarlos. Obviamente, se debe romper con el prototipo tradicional de empresa, en virtud del cual la empresa tiene que producir los mayores beneficios para los accionistas y dejar una mínima retribución al otro grupo de afectados. En efecto, desde los horizontes de una economía ética, debemos tener en cuentas no sólo los intereses grupales sino que la empresa debe perseguir intereses universalizables, que no beneficien a un grupo sino a todos por igual (Cortina, 2001b: 99).

Al respecto, Simon señala la importancia de que la empresa deportiva se responsabilice de todas las partes implicadas, que no sólo se refiere a los accionistas sino a todo el grupo de afectados («Stakeholders») (Simon, 2004: 185). El problema de la argumentación de Simon, es que no señala que esto debe hacerse en aras de los intereses universalizables 
que debe perseguir la empresa deportiva, aunque sí hable explícitamente de la responsabilidad social de la empresa deportiva.

En efecto, la empresa deportiva no puede hacer lo que le plazca y recurrir a medios ilícitos para aumentar sus beneficios, más bien deberá operar de acuerdo con principios razonables. De este modo Simon descarta de entrada que los accionistas de la empresa deportiva tengan el derecho de esperar obtener los mayores beneficios a costa de hacer cosas éticamente repugnantes. Se trata de que las empresas y las entidades corporativas relacionadas con el deporte, puedan y deban esperar obtener ganancias razonables dentro de los límites éticos.

Como he señalado, Simon plantea la comercialización del deporte en clave positiva, por lo que no es de extrañar que entienda como algo saludable para el deporte el que los eventos sean más entretenidos y divertidos. En efecto, si las competiciones deportivas son más entretenidas entonces habrá más demanda por parte de los espectadores y las ganancias aumentarán. De este modo, piensa Simon que se ven satisfechas las aspiraciones de los participantes que pagan por asistir a un encuentro deportivo que sea entretenido a la vez que divertido, y también se ven satisfechas las aspiraciones de los accionistas y demás grupos de afectados en el deporte, pues ven aumentados sus beneficios de manera lícita. Por eso nuestro autor considera que la deportividad puede ser entretenida porque demuestra las habilidades y virtudes humanas enfrentando los retos difíciles a la mente y el cuerpo en un beneficio mutuo y recíproco, en donde todas las partes implicadas salen beneficiadas.

Creo que Simon no hace frente a la cuestión de fondo, pues la responsabilidad social de la empresa deportiva no puede reducirse a un beneficio mutuo y recíproco en donde todos los afectados salen mutua y recíprocamente beneficiados. Es necesario plantear la empresa deportiva como empresa ética, interesada por alcanzar fines universalizables en lugar de fines que sólo satisfacen al mayor número de implicados, ya que esta posición nos acerca al utilitarismo ético antes que al universalismo ético. Esta perspectiva debe acompañar cualquier propuesta seria de ética del deporte, teniendo en cuenta las opiniones de los afectados, a los que debe tratarse como interlocutores válidos y promoviendo el consenso racional que les lleve a conseguir estos fines universalizables, que beneficien a todos y no a unos cuantos afectados.

Se trata, a mi modo de ver, de plantear la empresa deportiva como empresa ética, donde se aplique el principio ético al ámbito de la empresa deportiva considerando a cada miembro como interlocutor válido $\mathrm{y}$ teniendo en cuenta dialógicamente todos los intereses con la pretensión de hacerlos universalizables. Se trata, en consecuencia, de pasar de una 
cultura empresarial donde sólo se atiende a los intereses de los accionistas, una cultura de juegos de «suma cero» donde uno gana gracias a que otro pierda y que tiene malas consecuencias a corto y largo plazo, por una cultura de la cooperación y el mutuo entendimiento donde se tenga en cuenta los intereses de todos los afectados con proyección universal y no grupal.

En el caso de Simon, no queda claro si la empresa ética al ofertar los acontecimientos deportivos como eventos de mayor diversión que dejan satisfechos a los espectadores y a los grupos de afectados en la empresa deportiva - que ven incrementadas sus ganancias- sea la mejor forma de perseguir intereses universalizables, pues me da la sensación de que simplemente siguen persiguiendo intereses grupales y muy concretos, no universalizables. En efecto, la cuenta de resultados de la empresa ética, en nuestro caso la deportiva, deberá contemplar no sólo los resultados tangibles - como la obtención de ese beneficio mutuo- sino que también debe contemplar bienes intangibles y que son los que en última instancia propician la armonía y el trabajo cooperativo dentro de la empresa hasta asumir la responsabilidad social por el entorno desde la corresponsabilidad entre las relaciones internas, como señala muy acertadamente Cortina (Cortina, 2001b: 105).

En este punto quisiera dejar claro qué entiendo por empresa ética y qué marco debe producirse para que sea posible, para lo cual recurro de nuevo a la propuesta de Cortina en mi argumentación.

En primer lugar, la empresa ética no es desinteresada, pues persigue un interés o intereses universalizables, que es la satisfacción de los intereses de todos los afectados por una actividad, no sólo los grupales; en segundo lugar no debe guiarse por una ética de la convicción sino que debe asumir las consecuencias que pueden seguirse de la toma de decisiones para alcanzar la meta de la empresa. Se trata, a mi modo de ver, de una ética de la responsabilidad propia del deontologismo moderno - como el que propone Apel- que a diferencia del deontologismo kantiano, asume las consecuencias y subconsecuencias de las acciones que previamente todos los afectados (en tanto que empoderados) se han comprometido a asumir mediante un consenso; se trata de una empresa en donde se pide que la toma de decisiones sea moralmente correcta en la trama organizativa y previamente consensuada a través del diálogo intersubjetivo.

Para conseguir todo esto, es necesario un marco donde se desenvuelva la empresa ética. En primer lugar, deberá perseguir metas sociales por las que cobra su sentido en satisfacer las necesidades humanas. En el caso de la empresa deportiva, no sólo se tiene como meta social satisfacer las necesidades humanas de los grupos afectados 
- aunque muchos de ellos obtienen los medios económicos para vivir de su actividad en dicha empresa-, sino tener una proyección social más amplia trasmitiendo modelos de excelencia moral que sirvan de ejemplo para guiar el comportamiento de los demás miembros de la sociedad civil, pues en una sociedad donde los valores morales convencionales que servían de guía para los sujetos están en crisis, el deporte puede constituir el mejor vehículo de transmisión de los valores de convivencia, camaradería y respeto mutuo imprescindibles para una sociedad que quiera ser ética y responsable (Bento 2006 y 1990).

Pero para proveer este marco que haga posible una empresa ética también son necesarios unos mecanismos adecuados para ponerla en marcha. Aquí nos puede ayudar la economía de mercado, sin que la comercialización del deporte sea el fin de la empresa deportiva, aunque sí sea un medio que nos permita alcanzar el fin último del deporte. Necesitará también de un marco jurídico-político, sin que ello suponga perseguir los intereses marcados por posibles pactos sectoriales, ya que deben perseguir intereses universalizables. Y por último necesitaran de una moral crítica tan necesaria para no errar el camino convirtiendo lo que son medios en fines y los fines de la práctica en medios, o peor aún no teniéndolos en cuenta, arrojándolos al desuso.

\section{Conclusión: el «espíritu ético» en el deporte}

Después de ver cómo aborda Simon el problema de la comercialización del deporte desde su propuesta de ética de la competición, es bueno que saquemos algunos puntos en claro que ayuden en un futuro próximo a seguir trabajando en clave ética sobre los problemas que se siguen en la práctica del deporte contemporáneo, especialmente en el deporte de competición que se ha convertido en «deporte espectáculo».

Hemos aclarado varias cosas: primero, que el ideal de la ética de la competición deportiva no puede entenderse como «juego de suma cero», es decir, como aquella propuesta en donde ganan unos a costa de que los otros pierdan; segundo, que la ética de la competición deportiva se caracteriza por el trabajo cooperativo en la búsqueda recíproca de la excelencia moral, persiguiendo los valores morales internos a la práctica - como la disciplina, la dedicación o el esfuerzo cooperativo- siempre desde el respeto a las reglas constitutivas y a la zona de acción establecida por ella; $y$, tercero, que la propuesta de la ética de la competición no puede entenderse como una forma encubierta de autodesarrollo con la que Simon quisiera dar una especie de rodeo, para evitar afrontar el problema de fondo en deporte, evadiéndolos y cambiándolos por la idea 
de que en realidad la competición deportiva nos ayuda al autodesarrollo de la excelencia y del carácter de cada competidor.

Dicho esto, creo que estoy en condiciones de ofrecer algunas conclusiones.

En primer lugar, ha quedado claro que el problema ético de la comercialización del deporte puede afrontarse desde dos perspectivas dispares:

Primero, la de los partidarios de la «corruption thesis» que lo hacen desde un lado negativo, viéndola como algo a erradicar, pues atendiendo a motivaciones externas - como la ganancia económica- se pervierte la naturaleza de la práctica deportiva. La comercialización del deporte no sólo corrompe los fines de éste, sino que lo convierte en un instrumento para la consecución de los fines externos a la práctica deportiva. Hablar del poder corruptor que ejerce la comercialización en el deporte se refiere a la mala práctica de ciertas personas pertenecientes al mundo del deporte que guiados por fines lucrativos pervierten la práctica, pero no pervierten la naturaleza misma del deporte que se caracteriza por esa moral interna.

Segundo, la propuesta de Simon del todo novedosa, pues no se centra en el lado negativo de la comercialización, sino que más bien denuncia las prácticas de algunos de los implicados en la práctica del deporte -que deben entenderse como «Stakeholders», «grupos de afectados»- que en lugar de buscar los bienes internos en el deporte, sólo se interesan por los bienes externos atendiendo a motivaciones externas; por tanto lo malo de la comercialización del deporte está en el uso que se hace de ella cuando sólo se atiende a la persecución de bienes externos como la ganancia económica, la fama y similares.

Ambas posiciones aportan importantes núcleos de reflexión en torno al problema ético de la mercantilización de la práctica deportiva, aunque desde mi modesto punto de vista considero que el problema de la comercialización del deporte debe centrarse en otro orden de reflexión. Me refiero a la relación entre medios-fines.

En este sentido, el enfoque de una ética aristotélica puede contribuir muy positivamente a la erradicación del problema ético de la comercialización de la práctica deportiva, aunque considero que no debe cerrarse en el aristotelismo, sino abrirse a otras corrientes filosóficas como las deontológicas propias de las propuestas de éticas neo-kantianas elaboradas por Karl-Otto Apel o Jürgen Habermas.

Digo esto, pues una de las ventajas que plantea la ética aristotélica es la recuperación de importantes categorías y virtudes morales, que no han sabido tener en cuenta otras propuestas de ética más contemporánea. Así pues, para la moral aristotélica juega un importante papel la virtud de la 
prudencia. Para Aristóteles la prudencia no sólo comporta el conocimiento de los fines sino también el de los medios, por eso muchos de los estudiosos del Estagirita han querido ver que el conocimiento moral aristotélico implica esencialmente un doble acto de cognición que se centra en la relación fines-medios.

Esta doble distinción aristotélica del conocimiento moral entre finesmedios, puede ayudarnos a plantear la comercialización del deporte desde un enfoque ético que evite caer en posturas radicales como la de los partidarios de la «corruption thesis», que sólo la conciben desde un enfoque negativo, como algo que corrompe los ideales de la práctica deportiva. En efecto, pienso que la comercialización del deporte no presentaría ningún problema ético si se parte del hecho de que la ganancia económica en deporte no puede concebirse como el fin de éste, sino como un medio para su subsistencia, pues resulta evidente que cualquier club deportivo de élite debe costear desde el sueldo de sus deportistas, al sueldo de los entrenadores, personal de mantenimiento, etc.

Por tanto, la comercialización del deporte no presentaría mayores problemas si se la ve como un medio para cubrir todas las prestaciones que se derivan en un club deportivo. Otra cosa muy diferente es cuando los empresarios deportivos conciben la ganancia económica y la fama como el fin último del deporte, fomentando que se gane el mayor número de encuentros cuando ello redunda en mayores beneficios.

En definitiva, lo importante consiste en aceptar que ganar o aumentar los beneficios económicos no es necesariamente un signo de éxito competitivo y perder no es una muestra de fracaso, pues como dice el famoso lema de Pierre de Coubertein: «important thing in life is not victory, but struggle; the essential is not to have won but to have fought wells) (Andrew, 1998: 221). Solo con este «espíritu ético» lograremos que el horizonte ético florezca en la práctica del deporte.

\section{REFERENCIAS}

-Andrew, E. (1998). Sport, Ethics of. En R. Chadwick (Ed.), Encyclopedia of Applied Ethics (Vol. 4). San Diego: Academic Press.

-Apel, K.-O. (1998). Teoría de la verdad y ética del discurso. Barcelona: Paidós.

-Bento, J. O. (2006). Do desporto como um projecto ético para uma mudança nas mentalidades e atitudes. Pedagógia do desporto. Rio de Janeiro: Editora Guanabara Koogan.

-Bento, J. O. (1990). A procura de referencias para uma Ética do Desporto. Desporto, Ética e Sociedade. Actas. Porto: FCDEF/UP. 
-Conill, J. (2006). Ética hermenéutica. Madrid: Tecnos.

-Cortina, A. (2010). Justicia cordial. Madrid: Trotta.

-Cortina, A. (2001a). Ética sin moral. Madrid: Tecnos.

-Cortina, A. (2001b). Ciudadanos del mundo: hacia una teoría de la ciudadanía. Madrid: Tecnos.

-Drucker, P. F. (1998). La sociedad poscapitalista. Arganda del Rey: Ediciones Apóstrofe.

-Frankl, V. E. (1982). Deporte: ascetismo de hoy día. En V. E. Frankl, Psicoterapia y Humanismo: ¿Tiene un sentido la vida? Madrid: Fondo de Cultura Económica.

-MacIntyre, A. (1981). After virtue, Notre Dame, Ind.: University of Notre Dame Press.

-MacIntyre, A. (2001). Animales racionales y dependientes. Barcelona: Paidós.

-Michener, J. (1976). Sports in America. New York: Random House.

-Mieth, D. (1989). Ética del deporte. Concilium, 225, 241-255.

-Monan, J. D. (1959). The Doctrine of Moral Knowledge in Aristotle's Protepticus, Eudemiam and Nichomachen Ethics. Tesis Lovaina.

-Moncho, J. R. (1972). La unidad de la vida moral según Aristóteles. Valencia: Anales del Seminario de Valencia.

-Morgan, W. J. (1994). Leftist Theories of Sport: A Critique and Reconstruction. Chicago: University of IL Press.

-Simon, R. L. (1991). Fair Play: Sport, Values and Society. Boulder: Westview Press.

-Simon, R. L. (2004). Fair Play: The ethics of Sport. Boulder: Westview Press.

Sumario: 1. La ética de la competición deportiva; 2. La comercialización del deporte desde la ética de la competición de Robert L. Simon; 2.1. "Corruption thesis»: El lado negativo de la comercialización del deporte; 2.2. El lado positivo de la comercialización del deporte: la originalidad de Simon; 3. Responsabilidad social de la empresa deportiva; Conclusión: el «espíritu ético» en el deporte; Referencias. 